\title{
Prevalência de alterações ósseas no tarso de potros Crioulos de até vinte e seis meses de idade
}

\author{
Bone changes prevalence in the tarsus of Crioulo yearlings up to 26 months of age
}

\author{
Miguel Gallio $^{\text {I* }}$ Marcos da Silva Azevedo ${ }^{\mathrm{I}}$ Karin Erica Brass ${ }^{\mathrm{II}}$ \\ Flávio Desessards De La Corte ${ }^{\mathrm{II}}$ Luís Felipe Dias Lopes ${ }^{\mathrm{III}}$
}

\section{RESUMO}

\begin{abstract}
Com o objetivo de definir a prevalência de alterações ósseas no tarso, foram examinados radiograficamente 77 potros da raça Crioula, com até 26 meses de idade e a relação das lesões com parâmetros biométricos e de manejo. Os animais foram divididos em dois grupos por idade: G1, incluindo potros de nove a 18 meses (34/77); e G2, com animais de 19 a 26 meses (43/77). Os animais também foram divididos pelo tempo de preparo para exposição morfológica em GC, grupo controle (29/77), os animais sem preparo para exposição; e GI, grupo incentivo (48/77), animais com preparo médio de 4,55 meses, e foram separados por sexo em machos (46/77) e fêmeas (31/77). Os dados foram coletados em 24 propriedades de criação ou centros de treinamento/preparo do estado do Rio Grande do Sul. Lesões radiograficamente visíveis foram observadas em 79,2\% (61/77) dos animais, 80,4\% dos machos, 77,4\% das fêmeas, 77,3\% dos animais do GC, 79,2\% dos animais do GI, 86,7\% dos animais do G1 e 76,7\% dos animais do G2. O peso médio dos potros de 10 a 12 meses de idade foi $293,2 \mathrm{~kg}$, representando $71,3 \%$ do peso adulto; o peso médio dos animais com idade de 19 a 26 meses foi de $360,5 \mathrm{~kg}$ (87,6\% do peso adulto); do GC foi de $288,9 \mathrm{~kg}$ (70,2\% do peso adulto); e do GI $341,9 \mathrm{~kg}$ (83,1\% do peso adulto), apesar da idade média dos dois últimos grupos ter sido de apenas 16,8 e 19,0 meses, respectivamente. Observou-se relação positiva entre o aumento do escore corporal dos animais $(P=0,01 ; r=0,3)$, do tempo de preparo dos animais para exposição morfológica $(P=0,0001 ; r=0,7)$ e do escore de deposição de gordura na crista do pescoço $(P=0,02 ; r=0,3)$ com as lesões radiográficas observadas no tarso dos potros. Vários fatores podem estar envolvidos na osteoartrite társica distal, entretanto, em cavalos Crioulos, o fator mais importante presente nas criações foi o sobrepeso.
\end{abstract}

Palavras-chave: osteoartrite juvenil, sobrepeso, doença ortopédica do desenvolvimento, doença articular degenerativa.

\section{ABSTRACT}

Aiming to evaluate the prevalence of bone changes, radiographs were taken from both tarsal joints of 77 Crioulo yearlings, up to 26 months of age. The degree of changes was correlated with biometric parameters and management practices. The animals were separated into six groups, according to age: G1, including nine to 18 months old foals (34/77) and G2, with animals between 19 to 26 months of age (43/77); preparation time to halter class shows: GC, including animals who were not submitted to any preparation (29/77), and GI, consisting of young horses prepared to participate (average of 4.55 months of preparation time) in halter shows (48/77); and gender: colts (46/77) and fillies (31/77). Data were obtained from 24 breeding farms or training centers in Rio Grande do Sul state. Results showed that 79.22\% (61/77) of the animals presented some degree of radiographically visible lesions, being $80.4 \%$ of males, $77.4 \%$ of females, $77.3 \%$ in GC, $79.2 \%$ in GI, $86.7 \%$ in G1 and $76.7 \%$ in G2. The mean body weight of the 10 to 12 month old yearlings (G1) was $293.2 \mathrm{~kg}$, representing $71.3 \%$ of the breed's mature weight and that of the 19 to 26 month old (G2) animals was $360.5 \mathrm{~kg}$ ( $87.6 \%$ of the mature weight). The mean body weight of the GC was $288.9 \mathrm{~kg}$ (70.2\% of the mature weight) and of GI was $341.9 \mathrm{~kg}$ (83.1\% of the mature weight), although the average age of both groups was 16.8 and 19.0 months, respectively. A significant relationship was found between the animal's body condition score $(P=0.01 ; r=0.3)$, time of preparation to halter class shows $(P=0.0001 ; r=0.7)$, and neck crest score $(P=0.02$; $r=0.3$ ), and degree of the radiographic lesions found in the tarsi. Several factors can be involved in the development of distal tarsal juvenile osteoarthritis; however, in Crioulo breeding farms, the most important factor present was the horse's overweight.

Key words: juvenile osteoarthritis, overweight, developmental orthopaedic disease, degenerative joint disease.

\footnotetext{
'Programa de Pós-graduação em Medicina Veterinária, Universidade Federal de Santa Maria (UFSM), 97105-900, Santa Maria, RS, Brasil. E-mail: vetgallio@yahoo.com.br.*Autor para correspondência.

IIDepartamento de Clínica de Grandes Animais, Centro de Ciências Rurais (CCR), UFSM, Santa Maria, RS, Brasil.

IIIDepartamento de Ciências Administrativas, Centro de Ciências Sociais e Humanas (CCSH), UFSM, Santa Maria, RS, Brasil.
} 


\section{INTRODUÇÃO}

A utilização de cavalos cada vez mais jovens nas atividades atléticas e exposições, juntamente com a crescente valorização comercial da raça Crioula no Brasil, induzem os criadores a intensificar a criação desses animais. Alguns aspectos individuais do crescimento dos potros são ignorados, resultando, assim, na produção de um animal com percentuais de musculatura e gordura incompatíveis com sua idade e estrutura óssea (FREEMAN, 2005).

Este cenário de criação intensiva dos equinos pode levar ao surgimento de alterações musculoesqueléticas nos potros jovens, que são descritas na literatura como doenças ortopédicas do desenvolvimento (DODs). MCILWRAITH (2004) define as DODs como um conjunto de alterações clínicas que ocorrem na estrutura musculoesquelética de animais jovens, tais como osteocondrose (lesões de osteocondrose dissecante, lesões císticas subcondrais), displasia fiseal (epifisites), deformidades angulares e flexurais, alterações dos ossos cuboides do carpo/ tarso e as osteoartrites juvenis.

Segundo FREEMAN (2005), os três parâmetros que mais influenciam no crescimento dos animais são a genética, os programas de exercícios aos quais são submetidos e a nutrição. As DODs são mais comumente observadas em raças de crescimento rápido (Puro Sangue de Corrida, Quarto-de-Milha, Paint Horse, por exemplo) e tem, fundamentalmente, um caráter multifatorial. Os excessos nutricionais (ou a nutrição desbalanceada) e o trauma repetitivo estão entre os principais fatores que atuam negativamente sobre as cartilagens articulares, desencadeando as DODs (MCILWRAITH, 2004). O peso corporal excessivo, devido à alta taxa de gordura é o fator mais prejudicial à saúde das articulações.

BRANDT et al. (2008) destacam que a osteoartrite ou doença articular degenerativa (DAD) é considerada a alteração musculoesquelética crônica mais importante em equinos e humanos e resulta da incapacidade dos tecidos articulares se recuperarem do dano causado por estresse mecânico excessivo (força/unidade de área). A degeneração progressiva da cartilagem articular é característica na patogênese da osteoartrite, levando à perda da função articular e dor crônica debilitante. A DAD no tarso é a causa mais comum de claudicação em equinos e das claudicações associadas ao tarso. Sua ocorrência pode ser unilateral, porém a apresentação bilateral é mais frequente (DABAREINER et al., 2003).

Em potros, as osteoartrites das articulações intertársica distal (ITD) e tarsometatarsiana (TMT) são também consideradas manifestações clínicas das DODs (MCILWRAITH, 2004). Vários fatores, incluindo má conformação do membro (tarso valgus ou varus), tipo de atividade (adestramento, salto, rédeas, corridas de trote), ossificação incompleta dos ossos cuboides do carpo e tarso, com subsequente colapso, podem predispor ao aparecimento da osteoartrite nos potros (LATIMER, 2004).

Considerando o crescente número de potros jovens examinados por claudicação com origem no tarso, o objetivo deste trabalho foi definir a prevalência de alterações ósseas no tarso de potros Crioulos com até 26 meses de idade, ainda não domados e que tenham ou não participado de exposições morfológicas da raça Crioula. Também objetivou-se correlacionar os graus de lesões observados com o peso e escore corporal, idade, escore de deposição de gordura na crista do pescoço, altura, tempo de preparo para exposição morfológica e o período de exercício ao qual os animais eram submetidos.

\section{MATERIAL E MÉTODOS}

Foram examinados, em 24 centros criatórios ou de treinamento/preparo, 77 potros da raça Crioula, 31 fêmeas e 46 machos, sendo que 48 animais eram participantes das provas de incentivo da raça (categoria jovem nas provas de conformação) e 29 animais não estavam sendo preparados para exposições e foram alocados como grupo controle. A idade dos animais variou entre nove e 26 meses $(18,2 \pm 5,8)$. As imagens radiográficas dos 154 tarsos avaliados neste estudo foram produzidas utilizando um emissor portátil ${ }^{\mathrm{A}}$ com películas radiográficas ${ }^{\mathrm{B}}$ de 25,4cmx20,3cm em chassis rígido ${ }^{\mathrm{C}}$, compatível com tela intensificadora rápida. As articulações do tarso foram avaliadas radiologicamente usando as projeções lateromedial, dorsoplantar, dorsolateralplantaromedial obliquada e dorsomedialplantarolateral obliquada (VANDERPERREN et al., 2009).

As radiografias foram analisadas por dois examinadores experientes quanto à presença de alterações sugestivas de DODs sem o conhecimento sobre a que grupo pertenciam os animais. Especificamente, as lesões de DAD foram classificadas conforme MARANHÃO et al. (2006), que definem como grau 0: imagens radiográficas sem alterações, grau 1: presença de osteófitos intra ou periarticulares, grau 2: presença de osteófitos, diminuição do espaço articular e alterações na densidade do osso subcondral com ou sem lesões císticas, grau 3: presença de anquilose parcial e grau 4: presença de anquilose completa. 
No momento do exame radiográfico, também foram coletados dados referentes à alimentação, peso, idade e altura dos animais, escore corporal (EC) seguindo a escala de pontuação de 1-9, proposta por HENNEKE et al. (1983), escore de deposição de gordura na crista do pescoço (EGCP), seguindo escala de 0-5, proposta por CARTER et al. (2009). Também dados sobre o tempo (em meses) que os animais estavam sendo preparados para exposição morfológica (TPEM), que envolve o estabulamento dos animais e alimentação diferenciada, somado a um programa de exercício normalmente realizado em áreas circulares (redondel) com piso de areia solta, onde os animais são trotados tanto em sentido horário, quanto anti-horário por tempo variável.

Os dados obtidos foram analisados pelo programa Statistical Analisys System - SAS, versão 9. A correlação de Pearson $(r)$ foi estabelecida entre a ocorrência de lesão radiográfica visível (excetuandose os membros classificados como grau 0: sem lesão) com os parâmetros biométricos e de manejo dos animais. Estabeleceu-se o nível de significância $\mathrm{P}<0,05$. Posteriormente, os dados foram separados em grupos, sendo um correspondente aos animais de nove a 18 meses (G1) (34/77), outro grupo com animais de 19-26 meses (G2) (43/77), um grupo de fêmeas (31/77), um grupo de machos (46/77), um grupo com os animais controle (GC) (29/77) e um grupo com os potros participantes das categorias de incentivo da raça (GI) (48/77). Os dados foram novamente analisados seguindo o já proposto. Após, foram efetuadas as comparações de médias entre os grupos pelo teste de Mann-Whitney, com nível de significância $\mathrm{P}<0,05$.

\section{RESULTADOS}

Foi constatado que 79,2\% (61/77) dos animais apresentavam alguma alteração radiográfica visível no tarso compatível com lesões de osteoartrite das articulações ITD ou TMT. As fêmeas representaram 40,3\% (31/77) do total da amostra, sendo que 77,4\% (24/31) apresentaram alterações radiográficas. Os machos representaram 59,7\% (46/77) dos animais estudados e, destes, 80,4\% (37/46) apresentaram alterações radiográficas.

No total, foram avaliados 154 tarsos, dos quais $35,1 \%$ (54/154) foram classificados como grau 0 (sem lesão radiológica visível), 36,3\% (56/154) como grau $1,18,2 \%(28 / 154)$ como grau 2 e $10,4 \%$ (16/154) como graus 3 e 4 . Somente 20,8\% (16/77) dos animais não tinham sinais radiológicos de lesão no tarso e somente um animal apresentou claudicação relacionada às articulações estudadas. Um animal apresentou lesão compatível com osteocondrose dissecante (OCD) na tróclea lateral do tálus.

As médias dos parâmetros de idade, peso, altura, escore corporal (EC), escore de deposição de gordura na crista do pescoço (EGCP), grau de lesão radiográfica visível no tarso esquerdo (GLTE) e direito (GLTD) e tempo de preparo para exposição morfológica (TPEM) dos grupos G1, G2, machos, fêmeas, GI e GC encontram-se na tabela 1.

Nos grupos de machos e fêmeas, não foram encontradas diferenças estatísticas entre os parâmetros observados. Quando se avaliaram as médias dos grupos G1 e G2, constatou-se que a idade $(\mathrm{P}=0,0001)$, o peso corporal $(\mathrm{P}=0,001)$, a altura $(\mathrm{P}=0,001)$, a quantidade de ração concentrada fornecida aos animais $(\mathrm{P}=0,005)$ e o tempo em meses que os animais estavam em preparo para as exposições morfológicas $(\mathrm{P}=0,01)$ foram maiores nos animais do G2.

Com relação ao preparo (GI) ou não (GC) para exposição morfológica dos animais, o peso corporal $(\mathrm{P}=0,002)$, a altura dos animais $(\mathrm{P}=0,02)$, a quantidade de ração concentrada fornecida $(\mathrm{P}=0,001)$, o escore corporal dos animais $(\mathrm{P}=0,002)$,

Tabela 1 - Médias ( \pm DP) dos parâmetros avaliados em 77 potros Crioulos divididos nos grupos G1(09a 18 meses), G2 (19 a 26 meses), machos, fêmeas, GC (controle) e GI (participantes da categoria incentivo).

\begin{tabular}{|c|c|c|c|c|c|c|}
\hline Média & $\mathrm{G} 1(\mathrm{n}=34)$ & G2 $(n=43)$ & MACHOS $(n=46)$ & FÊMEAS $(n=31)$ & GC $(n=29)$ & GI $(n=48)$ \\
\hline Idade (meses) & $12,3^{\mathrm{a}}( \pm 2,9)$ & $22,9^{\mathrm{b}}( \pm 1,9)$ & $17,6( \pm 5,8)$ & $19,2( \pm 5,8)$ & $16,8( \pm 5,9)$ & $19,0( \pm 5,7)$ \\
\hline Peso (kg) & $273,7^{\mathrm{a}}( \pm 64,0)$ & $360,5^{\mathrm{b}}( \pm 60,5)$ & $314,8( \pm 54,2)$ & $332,2( \pm 99,8)$ & $288,9^{\mathrm{a}}( \pm 57,2)$ & $341,9^{\mathrm{b}}( \pm 78,7)$ \\
\hline Altura (cm) & $126,6^{\mathrm{a}}( \pm 6,3)$ & $134,9^{\mathrm{b}}( \pm 3,7)$ & $131,8( \pm 4,8)$ & $130,4( \pm 8,4)$ & $129^{\mathrm{a}}( \pm 6,5)$ & $132,7^{\mathrm{b}}( \pm 6,1)$ \\
\hline EC & $7,3( \pm 0,8)$ & $7,4( \pm 1,1)$ & $7,2( \pm 0,8)$ & $7,6( \pm 1,1)$ & $6,9^{\mathrm{a}}( \pm 0,8)$ & $7,6^{\mathrm{b}}( \pm 0,9)$ \\
\hline EGCP & $3,0( \pm 0,5)$ & $3,2( \pm 0,7)$ & $3,0( \pm 0,6)$ & $3,2( \pm 0,7)$ & $2,8^{\mathrm{a}}( \pm 0,6)$ & $3,3^{\mathrm{b}}( \pm 0,6)$ \\
\hline GLTD & $1,6( \pm 0,8)$ & $1,6( \pm 0,8)$ & $1,5( \pm 0,7)$ & $2,1( \pm 1,6)$ & $1,7( \pm 0,9)$ & $1,8( \pm 1,3)$ \\
\hline GLTE & $1,5( \pm 0,6)$ & $1,7( \pm 1,0)$ & $1,5( \pm 0,7)$ & $1,8( \pm 1,1)$ & $1,4( \pm 0,6)$ & $1,7( \pm 1,0)$ \\
\hline TPEM (meses) & $1,7^{\mathrm{a}}( \pm 2,2)$ & $3,7^{\mathrm{b}}( \pm 4,3)$ & $2,6( \pm 3,3)$ & $3,2( \pm 4,2)$ & $0,1^{\mathrm{a}}( \pm 0,3)$ & $4,5^{\mathrm{b}}( \pm 3,8)$ \\
\hline
\end{tabular}

${ }^{\mathrm{a}, \mathrm{b}}$ Valores com sobrescritos diferentes na mesma linha são estatisticamente diferentes. 
o escore de deposição de gordura na crista do pescoço $(p=0,001)$, o tempo de exercício a que os animais eram submetidos diariamente $(\mathrm{P}=0,001)$ e o tempo de preparo para exposição morfológica $(\mathrm{P}=0,001)$ foram maiores no GI, quando comparado ao GC.

Considerando o número total de animais, houve correlação positiva entre o grau de lesão radiográfica no tarso esquerdo e o escore corporal $(\mathrm{P}=0,01 ; \mathrm{r}=0,3)$, o escore de deposição de gordura na crista do pescoço $(\mathrm{P}=0,002 ; \mathrm{r}=0,3)$ e o grau de lesão radiográfica no tarso direito $(\mathrm{P}=0,03 ; \mathrm{r}=0,3)$. $\mathrm{O}$ grau de lesão radiográfica no tarso direito, por sua vez, teve correlação positiva com a idade $(\mathrm{P}=0,0001, \mathrm{r}=0,8)$ e o tempo de preparo para exposição morfológica $(\mathrm{P}=0,0001, \mathrm{r}=0,7)$, e correlação negativa com o peso corporal $(\mathrm{P}=0,03, \mathrm{r}=-0,3)$, a altura dos animais $(\mathrm{P}=0,0001, \mathrm{r}=-0,76)$ e o escore corporal $(\mathrm{P}=0,01$, $r=-0,3)$.

No GC, observou-se correlação positiva entre o grau de lesão radiográfica do tarso esquerdo e o escore corporal dos animais $(\mathrm{p}=0,01, \mathrm{r}=0,6)$ e o escore de deposição de gordura na crista do pescoço $(\mathrm{P}=0,03, \mathrm{r}=0,5)$. No GI, foi observada correlação positiva entre o grau de lesão radiográfica do tarso direito e a idade dos animais $(\mathrm{P}=0,0001, \mathrm{r}=0,8)$ e com o tempo de preparo para exposição morfológica que estes animais estavam submetidos $(\mathrm{P}=0,0001, \mathrm{r}=0,8)$. Percebeu-se também correlação negativa entre o grau de lesão radiográfica no tarso direito e o peso corporal $(\mathrm{P}=0,02, \mathrm{r}=-0,4)$, a altura dos animais $(\mathrm{P}=0,0001, \mathrm{r}=-0,8)$ e o escore corporal $(\mathrm{P}=0,002, \mathrm{r}=-0,5)$.

No G1, o grau de lesão radiográfica do tarso esquerdo se correlacionava positivamente com a idade dos animais $(\mathrm{P}=0,01, \mathrm{r}=0,6)$, o escore corporal $(\mathrm{P}=0,01, \mathrm{r}=0,5)$, o grau de lesão radiográfica no tarso direito $(\mathrm{P}=0,02, \mathrm{r}=0,6)$ e também tinha correlação negativa com o fornecimento de volumoso in natura ao animal $(\mathrm{P}=0,01, \mathrm{r}=-0,6)$.

No G2, foram encontradas correlações positivas entre o grau de lesão radiográfica do tarso esquerdo, a altura dos animais $(\mathrm{P}=0,04, \mathrm{r}=0,4)$ e o grau de lesão radiográfica do tarso direito $(\mathrm{P}=0,0001, \mathrm{r}=0,8)$.

Nos machos, encontrou-se correlação positiva entre grau de lesão radiográfica do tarso esquerdo e o escore corporal $(\mathrm{P}=0,005, \mathrm{r}=0,5)$, o escore de deposição de gordura na crista do pescoço $(\mathrm{P}=0,03, \mathrm{r}=0,4)$ e o grau de lesão radiográfica no tarso direito $(\mathrm{P}=0,007, \mathrm{r}=0,5)$. Também foi encontrada correlação negativa com a quantidade de alimento volumoso in natura fornecida $(\mathrm{P}=0,008, \mathrm{r}=-0,5)$.

Nas fêmeas, encontrou-se correlação positiva entre o grau de lesão radiográfica no tarso direito e a idade $(\mathrm{P}=0,0001, \mathrm{r}=0,9)$ e o período de preparo para exposição morfológica a que os animais eram submetidos $(\mathrm{P}=0,0001, \mathrm{r}=0,9)$. Além disso, encontrou-se correlação negativa entre o grau de lesão radiográfica no tarso direito e a altura dos animais $(\mathrm{P}=0,0001, \mathrm{r}=-0,9)$ e o escore corporal $(\mathrm{P}=0,01, \mathrm{r}=-0,5)$.

\section{DISCUSSÃO}

O exame radiográfico é a modalidade de diagnóstico por imagem mais comumente empregada na avaliação de DAD em equinos. A maioria dos potros da raça Crioula $(79,2 \%)$ deste estudo apresentaram lesões radiográficas compatíveis com DAD de grau variado em pelo menos um dos membros, sendo que as alterações radiográficas mais comumente encontradas foram osteófitos e/ou entesófitos periarticulares, lise do osso subcondral e/ou esclerose, irregularidades e estreitamento do espaço articular, lesões estas que VERSCHOOTEN\& SCHRAMME (1994) consideram comuns na avaliação dos casos de osteoartrite nas articulações distais do tarso. Esse achado mostra a alta prevalência desta patologia, atribuída ao elevado escore corporal dos animais deste estudo.

GARCIA et al. (2009) relataram que 83,3\% (25/30) dos potros da raça Mangalarga Marchador em preparo para exposição apresentavam osteoartrite társica, e atribuíram sua ocorrência ao excesso de exercício em terreno íngreme, que submete as articulações a trauma excessivo. Já no presente trabalho, o fator predisponente mais frequentemente observado foi a excessiva deposição de gordura, traduzida pelo aumento no escore corporal e de deposição de gordura na crista do pescoço dos animais que, segundo FREEMAN (2005), são dos fatores mais estressantes às articulações. Doenças articulares podem ser desencadeadas por alterações nutricionais, comprovadas por SAVAGE et al. (1993), ao induzirem o desenvolvimento de osteocondrose em potros sem raça definida através de dieta com $129 \%$ das necessidades energéticas recomendadas pelo Nacional Research Council (NRC).

FREEMAN (2005) cita que, para diminuir a ocorrência de DODs, incluindo a osteoartrite juvenil, os potros, aos 12 meses de idade, devem pesar em torno de $50-60 \%$ do peso adulto, que é de aproximadamente 411,4kg (CANTO et al., 2006). No presente estudo, os animais do G1 apresentavam em média 71,3\% (média=293,3kg) do peso corporal de um cavalo Crioulo adulto, fato este que pode estar relacionado à alta ocorrência de lesões articulares observadas nesses animais. 
Não foram encontradas diferenças nos graus de lesão radiográfica nos tarsos entre os animais do GC e GI. Esse é um dado diferente do esperado, uma vez que é sabido que o confinamento dos animais, juntamente com o preparo deles para exposições, normalmente, acarreta em fornecimento de alimentação com excesso calórico. Dessa forma, acredita-se que a pastagem possa ser um fator que leve à obesidade em cavalos crioulos, visto que, muitas vezes, o conteúdo nutricional da pastagem é subestimado, levando a erros de suplementação dos potros, o que resulta em uma taxa de crescimento excessivo (LAWRENCE\& PAGAN, 2005).

A dieta fornecida em excesso aos animais em crescimento é considerada o principal fator desencadeante das DODs (MCILWRAITH, 2004; FREEMAN, 2005). As principais consequências observadas devido ao manejo alimentar dos potros foram o escore corporal e deposição de gordura na crista do pescoço elevados como sinais de obesidade, com médias maiores nas fêmeas, fato este que pode ser atribuído à maior média de idade apresentada pelas fêmeas.

DABAREINER et al. (2003) afirmam que existe uma tendência das lesões de osteoartrite do tarso serem bilaterais. De forma semelhante, neste estudo, as correlações observadas quando se analisaram os dados de todos os animais, nos grupos de machos, G1 e G2, o aumento do grau das lesões radiográficas no tarso esquerdo e direito (geral: $\mathrm{P}=0,03, \mathrm{r}=0,3$; machos: $\mathrm{P}=0,007 ; \mathrm{r}=0,5$; G1: $\mathrm{P}=0,002 ; \mathrm{r}=0,6 ; \mathrm{G} 2: \mathrm{P}=0,0001, \mathrm{r}=0,8)$ foram positivas. A ocorrência de lesões bilaterais supõe-se estar relacionada com a patogenia da lesão, em que se culpam os movimentos de compressão e rotação do membro (DABAREINER et al., 2003), também a sobrecarga sobre o membro sadio como resposta à dor presente no membro inicialmente afetado, porém esta patogenia ainda não está comprovada e autores também relatam que a predisposição pode ser herdada geneticamente (BARNEVELD, 1996).

Uma vez que as doenças articulares degenerativas (DAD) são mais frequentes em animais adultos (LATIMER, 2004), é provável que a vida atlética dos potros avaliados neste estudo seja afetada negativamente a partir do momento em que a intensidade dos treinamentos aumentar, uma vez que as lesões tendem a se agravar com o tempo e o esforço colocado sobre as articulações envolvidas.

A alta incidência de lesões (79,2\%) identificada neste estudo deve servir de alerta a criadores e aos demais profissionais ligados à criação de cavalos Crioulos. Deve-se atentar para a importância da adoção de novas estratégias de alimentação e manejo dos animais durante o período de seu desenvolvimento, para que esses não comprometam seu futuro. Sabe-se que o período da desmama até chegar aos 12 meses de idade é o momento mais importante para o animal, no que diz respeito à gestão nutricional, para evitar as DODs (FREEMAN, 2005). Portanto, os potros desmamados devem ser suplementados adequadamente, dentro de uma expectativa de crescimento em ritmo moderado para a raça.

\section{CONCLUSÃO}

Potros Crioulos de até vinte e seis meses de idade apresentam elevada prevalência de sinais radiográficos compatíveis com osteoartrite társica. As alterações correlacionaram-se positivamente com o aumento do escore corporal, de deposição de gordura na crista do pescoço, com o aumento da idade e do tempo de preparo dos animais.

\section{COMITÊ DE ÉTICA}

Este trabalho foi aprovado pela Comissão de Ética no Uso de Animais-UFSM sob o parecer número 104/2011.

\section{AGRADECIMENTOS}

Agradeço ao Conselho Nacional de Desenvolvimento Científico e Tecnológico (CNPq) pelo financiamento da bolsa de estudos que tornou possível a realização deste trabalho e a Financiadora de Estudos e Projetos (FINEP), financiadora do equipamento utilizado neste estudo.

\section{FONTES DE AQUISIÇÃO}

${ }^{\text {A}}$ BOWIE $^{\circledR}$ ULTRALITE $10040 \mathrm{HF}$, EUA.

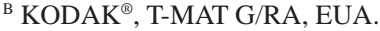

${ }^{\mathrm{C} O R T H O}$ REGULAR CURIX ${ }^{\circledR}$ screens, EUA.

\section{REFERÊNCIAS}

BARNEVELD, A. The role of breeding in sports performance and health with special emphasis in lameness prevention. Pferdeheilkunde, v.12, n.4, p.689-692. 1996. Disponível em: <http://www.pferdeheilkunde.de/>. Acesso em: 10 jun. 2011.

BRANDT, K.D., et al. Etiopathogenesis of osteoarthritis. Rheumatic Disease Clinics of North America, v.34, p.531-559, 2008. Disponível em: <http://www.sciencedirect.com/science/ article/pii/S0889857X08000458>. Acesso em: 10 jun. 2011. doi: 10.1016/j.rdc.2008.05.011.

CANTO, L.S. et al.Frequência de problemas de equilíbrio nos cascos de cavalos crioulos em treinamento. Brazilian Journal of Veterinary Research and Animal Science, v.43, n.4, p.489-495, 2006. . Disponível em: <http://www.revistasusp.sibi.usp.br/scielo. 
php?script=sci_arttext $\&$ pid=S1413-95962006000400008\&lng=pt \&nrm=iso $>$.Acesso em: 11 jun. 2012.

CARTER, R.A. et al. Apparent adiposity assessed by standardized scoring systems and morphometric measurements in horses and ponies. Veterinary Journal. v.179, p.204-210, 2009 Disponível em: <http://www.sciencedirect.com/science/article/pii/ S109002330800110X>.Acesso em: 11 jun. 2012. doi: 10.1016/j. tvjl.2008.02.29.

DABAREINER, R.M. et al. The tarsus. In: ROSS, M.W.; DYSSON, S.J. Diagnosis and management of lameness in the horse. St. Louis. Saunders, 2003. p.440-443.

FREEMAN, D. Managing young horses for sound growth Oklahoma, USA. Oklahoma Cooperative Extension Service, 2005. (ANSI, 3977). Disponível em: <http://pods.dasnr.okstate. edu/docushare/dsweb/Get/Document-2088/ANSI-3977web.pdf>. Acesso em: 25 abr. 2012.

GARCIA, R.S. et al.Estudo clínico e radiográfico da osteoartrite társica juvenil em potros da raça manga-larga marchador. Ciência Animal Brasileira, v.10, n.1, p.254-260, 2009. Disponível em: <www.revistas.ufg.br/index.php/vet/article/download/2316/4592>. Acesso em: 12 jun. 2012.

HENNEKE, D.R. et al. Relationship between condition score, physical measurements and body fat percentage in mares. Equine Veterinary Journal, v.15, n.4, p.371-372. 1983. Disponível em: <http://onlinelibrary.wiley.com/doi/10.1111/evj.1983.15.issue-4/i ssuetoc>. Acesso em: 12 jun. 2012. doi: 10.1111/j.2042-3306.1983. tb01826.x.

LATIMER, F.G. Tarsus and stifle. In: HINCHCLIFF, K.W.et al. Equine sports medicine and surgery. Saint Louis: Saunders, 2004. p.368-411.

LAWRENCE, L.A.; PAGAN, J.D. The role of nutrition in developmental orthopedic disease in the equine. In: MID-
ATLANTIC NUTRICION CONFERENCE, 3, 2005, Timonium, USA. Proceedings... Timonium: MANC, 2005.p.185-196.Acesso em: 10 jun. 2012. Online. Disponível em:<http://manc.umd.edu/ documents/2005_Complete_Proceedings.pdf $>$.

MARANHÃO, R.P.A. et al. Afecções mais frequentes do aparelho locomotor dos equídeos de tração no município de Belo Horizonte. Arquivo Brasileiro de Medicina Veterinária e Zootecnia. v.58, p.21-27, 2006. Disponível em: <http://www. scielo.br/pdf/abmvz/v58n1/28775.pdf $>$. Acesso em: 11 jun. 2012.

MCILWRAITH, C.W. Developmental orthopedic disease: problems of limbs in young horses. Journal of Equine Veterinary Science, v.24, n.11, p.475-479, 2004. Disponível em: <http:// top25.sciencedirect.com/subject/veterinary-science-andveterinary-medicine/24/journal/journal-of-equine-veterinaryscience/07370806/archive/11>. Acesso em: 10 abr. 2011. doi: 10.1016/j.jevs.2004.10.004

SAVAGE, C.J. et al. Effects of dietary energy and protein on induction of dyschondroplasia in foals. Equine Veterinary Journal, v.16, suppl.16, p.74-79, 1993. Disponível em: <http:// onlinelibrary.wiley.com/doi/10.1111/j.2042-3306.1993.tb04858.x/ abstract>. Acesso em: 01 fev. 2013. doi: 10.1111/j.2042-3306.1993. tb04858.x.

VANDERPERREN, K. et al. Diagnostic imaging of the equine tarsal region using radiography and ultrasonography. Part 2: Bony disorders. Veterinary Journal, v.179, p.188-196, 2009. Disponível em: <http://www.sciencedirect.com/science/article/pii/ S1090023307003115>. Acesso em: 20 abr. 2012. doi: 10.1016/j. tvjl.2007.08.025.

VERSCHOOTEN, F.; SCHRAMME, M. Radiological examination of the tarsus. Equine Veterinary Education, v.6, n.6, p.323-332, 1994. Disponível em: <http://onlinelibrary.wiley.com/ doi/10.1111/j.2042-3292.1994.tb01165.x/abstract>. Acesso em: 14 jun. 2012. doi: 10.1111/j.2042-3292.1994.tb01165.x. 